\title{
A TRANSITIVITY THEOREM FOR ALGEBRAS OF ELEMENTARY OPERATORS
}

\author{
BOJAN MAGAJNA
}

(Communicated by Palle E. T. Jorgensen)

\begin{abstract}
Let $\mathscr{A}$ be a $C^{*}$-algebra and $\mathscr{E}$ the algebra of all elementary operators on $\mathscr{A}$, and let $\vec{a}=\left(a_{1}, \ldots, a_{n}\right), \vec{b}=\left(b_{1}, \ldots, b_{n}\right) \in \mathscr{A}^{n}$. It is proved that $\vec{b}$ is contained in the closure of the set $\left\{\left(E a_{1}, \ldots, E a_{n}\right): E \in \mathscr{E}\right\}$ if and only if each complex linear combination $\sum_{j=1}^{n} \lambda_{j} b_{j}$ is contained in the closed two-sided ideal generated by $\sum_{j=1}^{n} \lambda_{j} a_{j}$. In particular, a bounded linear operator on $\mathscr{A}$ preserves all closed two-sided ideals if and only if it is in the strong closure of $\mathscr{E}$.
\end{abstract}

\section{INTRODUCTION, MOTIVATION, AND NOTATION}

An elementary operator on a ring $\mathscr{A}$ is a map $E: \mathscr{A} \rightarrow \mathscr{A}$ of the form

$$
E x=\sum_{i=1}^{m} u_{i} x v_{i} \quad(x \in \mathscr{A}),
$$

where $\vec{u}=\left(u_{1}, \ldots, u_{m}\right)$ and $\vec{v}=\left(v_{1}, \ldots, v_{m}\right)$ are fixed $m$-tuples of elements of $\mathscr{A}$. In the last decade there has been considerable interest in such operators, especially in the cases when $\mathscr{A}$ is the algebra $\mathscr{B}(\mathscr{H})$ of all bounded operators on a Hilbert space $\mathscr{H}$, the Calkin algebra or a general prime $C^{*}$-algebra (see [1, $7,8]$ and their bibliographies). In this note we will be concerned with algebras of elementary operators on general $C^{*}$-algebras.

Our study here is motivated by the following classical algebraic considerations. For any unital algebra $\mathscr{A}$ (over some field) the set $\mathscr{E}$ of all elementary operators on $\mathscr{A}$ is again an algebra (with the usual operations of addition, multiplication by scalars, and composition of operators). The algebra $\mathscr{A}$ itself can be regarded as a (left) module over $\mathscr{E}$, the submodules of which are precisely the two-sided ideals of $\mathscr{A}$, and the module endomorphisms of $\mathscr{A}$ are just the multiplications by elements of the centre $\mathscr{C}$ of $\mathscr{A}$. For any positive integer $n$ the direct sum $\mathscr{A}^{n}$ of $n$ copies of $\mathscr{A}$ is then, of course, also an $\mathscr{E}$-module. Let us consider the following question.

Given $\vec{a}, \vec{b} \in \mathscr{A}^{n}$, under what conditions does there exist an elementary operator $E \in \mathscr{E}$ such that $E \vec{a}=\vec{b}$ (that is, $E a_{j}=b_{j}$ for $j=1, \ldots, n$ )?

Received by the editors August 16, 1991.

1991 Mathematics Subject Classification. Primary 47D99, 46L05; Secondary 47B47.

Key words and phrases. Elementary operators, $C^{*}$-algebra, von Neumann algebra. 
One necessary condition is obvious: since $E a_{j}=b_{j}(j=1, \ldots, n)$ implies that $E\left(\sum_{j=1}^{n} \lambda_{j} a_{j}\right)=\sum_{j=1}^{n} \lambda_{j} b_{j}$ for arbitrary central elements $\lambda_{j} \in \mathscr{A}$, we see that $\sum_{j=1}^{n} \lambda_{j} b_{j}$ must be in the two-sided ideal generated by $\sum_{j=1}^{n} \lambda_{j} a_{j}$. To shorten the notation, for every $\vec{\lambda}=\left(\lambda_{1}, \ldots, \lambda_{n}\right) \in \mathscr{C}^{n}$ and every $\vec{x}=$ $\left(x_{1}, \ldots, x_{n}\right)$ the linear combination $\sum_{j=1}^{n} \lambda_{j} x_{j}$ will be denoted by $\vec{\lambda} \cdot \vec{x}$. Also, let us agree that the word 'ideal' means a two-sided ideal and, for each $x \in \mathscr{A}$, denote by $\langle x\rangle$ the ideal generated by $x$. We may now ask the following

Question 1. Is the condition that $\vec{\lambda} \cdot \vec{b} \in\langle\vec{\lambda} \cdot \vec{a}\rangle$ for each $\vec{\lambda} \in \mathscr{C}^{n}$ also sufficient for the existence of an elementary operator $E$ on $\mathscr{A}$ such that $E \vec{a}=\vec{b}$ ?

In the special case, when $\mathscr{A}$ has no nontrivial ideals, the answer to the last question is affirmative. Namely, in this case the condition reduces to the requirement that $\vec{\lambda} \cdot \vec{a}=0$ implies $\vec{\lambda} \cdot \vec{b}=0$ (for each $\vec{\lambda} \in \mathscr{C}^{n}$ ), and since $\mathscr{A}$ is a simple $\mathscr{E}$-module, the existence of $E \in \mathscr{E}$ satisfying $E \vec{a}=\vec{b}$ follows from the Jacobson density theorem [11, p. 220]. In general, however, the answer to Question 1 is negative, as is shown by the following example.

Example. Let $\mathscr{H}$ be an infinite-dimensional (complex) vector space, $\mathscr{L}$ the algebra of all linear operators on $\mathscr{H}, \mathscr{F}$ the ideal of all finite rank operators, and $c \in \mathscr{L}$ a fixed operator such that $c \notin \mathscr{F}$ and $c^{2} \in \mathscr{F}$. Let $\mathscr{A}$ be the subalgebra of $\mathscr{L}$ generated by $\mathscr{F}, c$, and the identity operator 1 , and put $\vec{a}=(1, c), \vec{b}=\left(1, c^{2}\right)$. Then clearly the centre of $\mathscr{A}$ consists of scalars only, and it is easy to verify that the only proper ideals in $\mathscr{A}$ are $0, \mathscr{F}$, and the ideal generated by $\mathscr{F} \cup\{c\}$. It follows that, with $\vec{\lambda}=\left(\lambda_{1}, \lambda_{2}\right) \in \mathscr{C}^{2}$, the ideal $\langle\vec{\lambda} \cdot \vec{a}\rangle=\left\langle\lambda_{1}+\lambda_{2} c\right\rangle$ is proper only if $\lambda_{1}=0$, and the condition $\vec{\lambda} \cdot \vec{b} \subset\langle\vec{\lambda} \cdot \vec{a}\rangle$ is satisfied for all $\vec{\lambda} \in \mathscr{C}^{2}$. On the other hand, we shall now see that there is no elementary operator $E$ on $\mathscr{A}$ such that $E \vec{a}=\vec{b}$.

Assume to the contrary, that there exist $u_{i}, v_{i} \in \mathscr{A} \quad(i=1, \ldots, m)$ such that

$$
\sum_{i=1}^{m} u_{i} 1 v_{i}=1 \text { and } \sum_{i=1}^{m} u_{i} c v_{i}=c^{2}
$$

Since the quotient algebra $\mathscr{A} / \mathscr{F}$ is obviously commutative, the last two identities imply that $c^{2}=c(\bmod \mathscr{F})$, but this is in contradiction with the fact that $c \notin \mathscr{F}, c^{2} \in \mathscr{F}$.

We are now going to say a few words about the analogy of the above purely algebraic question in the context of Banach algebras. Let $\mathscr{A}$ be a complex unital Banach algebra, and denote by $[x]$ the closed ideal generated by an element $x \in \mathscr{A}$. Given a positive integer $n$ and $\vec{a}, \vec{b} \in \mathscr{A}^{n}$ we may now ask Question 2. When does there exist a sequence of elementary operators $E_{k} \quad(k=$ $1,2, \ldots)$ on $\mathscr{A}$ such that $E_{k} \vec{a}$ converge to $\vec{b}$ ?

Obviously a necessary condition for the existence of such a sequence of elementary operators is that $\vec{\lambda} \cdot \vec{b} \in[\vec{\lambda} \cdot \vec{a}]$ for each $\vec{\lambda} \in \mathscr{C}^{n}$, but a simple modification of the example shows that this condition is not sufficient. (Namely, in the Example we replace $\mathscr{L}$ by the algebra $\mathscr{B}(\mathscr{H})$ of all bounded linear operators on a separable Hilbert space $\mathscr{H}$, and $\mathscr{F}$ by the ideal $\mathscr{K}(\mathscr{H})$ of all 
compact operators; we choose $c \in \mathscr{B}(\mathscr{H}) \backslash \mathscr{K}(\mathscr{H})$ so that $c^{2} \in \mathscr{K}(\mathscr{H})$, and let $\mathscr{A}$ be the Banach algebra generated by $\mathscr{K}(\mathscr{H}), c$, and the identity operator.) Concerning general Banach algebras, we state here only one simple result.

Proposition 1.1. Let $\mathscr{A}$ be a complex unital Banach algebra, and assume that $\vec{a}=\left(a_{1}, \ldots, a_{n}\right) \in \mathscr{A}^{n}$ is such that $[\vec{\lambda} \cdot \vec{a}]=\mathscr{A}$ for every $\vec{\lambda} \neq 0, \vec{\lambda} \in \mathbb{C}^{n}$. Then for every $\vec{b}=\left(b_{1}, \ldots, b_{n}\right) \in \mathscr{A}^{n}$ there exists an elementary operator $E$ on $\mathscr{A}$ such that $E \vec{a}=\vec{b}$.

Proof. The proof is by an induction on $n$. The case $n=1$ is trivial, so let $n>1$. We must prove that $\mathscr{E} \vec{a}=\mathscr{A}^{n}$, and for this it suffices to prove that there exists $F_{n}=F \in \mathscr{E}$ satisfying

$$
F a_{j}=0 \text { for } j=1, \ldots, n-1 \text { and }\left\langle F a_{n}\right\rangle=\mathscr{A} \text {. }
$$

For then, by the same argument, there exists for each $i=1, \ldots, n$ an $F_{i} \in \mathscr{E}$ satisfying $F_{i} a_{j}=0$ for $j \neq i$ and $\left\langle F_{i} a_{i}\right\rangle=\mathscr{A}$, and choosing $E_{i} \in \mathscr{E}$ so that $E_{i} F_{i} a_{i}=b_{i}$ (which is possible, since $\left\langle F_{i} a_{i}\right\rangle=\mathscr{A}$ ), we see that the operator $E \stackrel{\text { def }}{=} \sum_{i=1}^{n} E_{i} F_{i}$ then satisfies $E \vec{a}=\vec{b}$. To prove the existence of $F$ satisfying (1), suppose, to the contrary, that no such $F$ exists, and denote by $\mathscr{N}$ the left ideal in $\mathscr{E}$ consisting of all $F \in \mathscr{E}$ that satisfy $F\left(a_{1}, \ldots, a_{n-1}\right)=0$. Note that $\mathscr{N} a_{n}$ is an $\mathscr{E}$-submodule of $\mathscr{A}$ and hence, an ideal in $\mathscr{A}$. Since by assumption no $F \in \mathscr{E}$ satisfies (1), $1 \notin \mathscr{N} a_{n}$; therefore, $\mathscr{N} a_{n}$ is contained in some proper maximal ideal $\mathscr{M}$ of $\mathscr{A}$. By the induction hypothesis we have $\mathscr{E}\left(a_{1}, \ldots, a_{n-1}\right)=\mathscr{A}^{n-1}$. Since $\mathscr{N} a_{n} \subseteq \mathscr{M}$, the map

$$
\varphi: \mathscr{A}^{n-1} \rightarrow \mathscr{A} / \mathscr{M}, \quad \varphi\left(F a_{1}, \ldots, F a_{n-1}\right) \stackrel{\text { def }}{=} F a_{n}+\mathscr{M} \quad(F \in \mathscr{E})
$$

is a well-defined homomorphism of $\mathscr{E}$-modules. Let $\varphi_{1}, \ldots, \varphi_{n-1}$ be the components of $\varphi$ (that is, $\varphi_{i}: \mathscr{A} \rightarrow \mathscr{A} / \mathscr{M}$ are $\mathscr{E}$-module homomorphisms such that $\varphi\left(x_{1}, \ldots, x_{n-1}\right)=\sum_{j=1}^{n-1} \varphi_{j}\left(x_{j}\right)$ for each $\left.\left(x_{1}, \ldots, x_{n-1}\right) \in \mathscr{A}^{n-1}\right)$. For each $j$ the kernel of $\varphi_{j}$ must contain $\mathscr{M}$ (since for each $m \in \mathscr{M}$ we have $\varphi_{j}(m)=\varphi_{j}(m 1)=m \varphi_{j}(1)=0$ in $\left.\mathscr{A} / \mathscr{M}\right)$. Hence $\varphi_{j}$ induces an endomorphism $\lambda_{j}$ of the $\mathscr{E}$-module $\mathscr{A} / \mathscr{M}$. Such an endomorphism is necessarily a multiplication by a central element of $\mathscr{A} / \mathscr{M}$, but the centre of each simple algebra is a field and the only field among the complex Banach algebras is the field $\mathbb{C}$ of all complex numbers. It follows that $\lambda_{j} \in \mathbb{C}$ for each $j$. By the definition of $\varphi$ we now have

$$
a_{n}+\mathscr{M}=\varphi\left(a_{1}, \ldots, a_{n-1}\right)=\sum_{j=1}^{n-1} \lambda_{j} a_{j}+\mathscr{M} .
$$

Hence $a_{n}-\sum_{j=1}^{n-1} \lambda_{j} a_{j} \in \mathscr{M}$, but this contradicts the assumption that $[\vec{\lambda} \cdot \vec{a}]=\mathscr{A}$ for each nonzero $\vec{\lambda} \in \mathbb{C}^{n}$.

In the remaining part of this note we confine our attention to $C^{*}$-algebras, where the necessary condition that $\vec{\lambda} \cdot \vec{b} \in[\vec{\lambda} \cdot \vec{a}]$ for each $\vec{\lambda} \in \mathbb{C}^{n}$ turns out to be sufficient also for $\vec{b}$ to be in the norm closure of the set $\mathscr{E} \vec{a}$. At the same time the analogous question in the context of von Neumann algebras is answered. 
At the end we shall also consider the so-called range inclusion problem for elementary operators on factors.

\section{THE CASE OF $C^{*}$-ALGEBRAS}

Theorem 2.1. Let $\mathscr{A}$ be a $C^{*}$-algebra, $\mathscr{E}$ the algebra of all elementary operators on $\mathscr{A}, n$ a positive integer, and $\vec{a}, \vec{b} \in \mathscr{A}^{n}$. Then $\vec{b}$ belongs to the norm closure $\overline{\overline{\mathscr{E}} \vec{a}}$ of $\mathscr{E} \vec{a}$ if and only if for each $\vec{\lambda} \in \mathbb{C}^{n}$ the element $\vec{\lambda} \cdot \vec{b}$ is contained in the closed ideal $[\vec{\lambda} \cdot \vec{a}]$ generated by $\vec{\lambda} \cdot \vec{a}$.

If $\mathscr{A}$ is a von Neumann algebra, then $\vec{b}$ belongs to the closure $\overline{\mathscr{E}} \vec{a}$ of $\mathscr{E} \vec{a}$ in the weak operator topology of $\mathscr{A}^{n}$ if and only if for each $\vec{\lambda} \in \mathscr{C}^{n}$ (where $\mathscr{C}$ is the centre of $\mathscr{A}$ ) the relation $\vec{\lambda} \cdot \vec{a}=0$ implies $\vec{\lambda} \cdot \vec{b}=0$.

For a von Neumann algebra $\mathscr{R} \subseteq \mathscr{B}(\mathscr{H})$ we denote by $M_{m, n}(\mathscr{R})$ the set of all $m \times n$ matrices with entries from $\mathscr{R}$, and we let $M_{n}(\mathscr{R})=M_{n, n}(\mathscr{R})$. Further, we identify $\mathscr{R}^{n}$ with $M_{n, 1}(\mathscr{R})$ so that elements of $\mathscr{R}^{n}$ are regarded as operators from $\mathscr{H}$ to $\mathscr{H}^{n}$. To prove Theorem 2.1 we first need a generalization of the well-known fact that every weakly closed ideal in a von Neumann algebra is generated by a central projection [4, p. 443].

Lemma 2.2. Let $\mathscr{R}$ be a von Neumann algebra acting on a Hilbert space $\mathscr{H}$. Then for each right $\mathscr{R}$-submodule $\mathscr{M}$ in $\mathscr{R}^{n}$ which is closed in the weak operator topology there exists an idempotent $p \in M_{n}(\mathscr{R})$ such that $\mathscr{M}=p \mathscr{R}^{n}$. Moreover, if $\mathscr{M}$ is an $\mathscr{R}$-bimodule, then $p \in M_{n}(\mathscr{C})$, where $\mathscr{C}$ is the centre of $\mathscr{R}$.

Proof. Since $\mathscr{M}$ is a right $\mathscr{R}$-module, we have

$$
\mathscr{M}=\mathscr{M} \mathscr{R}=\mathscr{M} M_{1, n}(\mathscr{R}) M_{n, 1}(\mathscr{R}) .
$$

Observe that $\mathscr{M} M_{1, n}(\mathscr{R})$ is a right ideal in the von Neumann algebra $M_{n}(\mathscr{R})$. Hence there exists a projection $p \in M_{n}(\mathscr{R})$ such that $\overline{\mathscr{M} M_{1, n}(\mathscr{R})}=p M_{n}(\mathscr{R})$. It follows that

$$
\mathscr{M}=\mathscr{M} M_{1, n}(\mathscr{R}) M_{n, 1}(\mathscr{R}) \subseteq p M_{n}(\mathscr{R}) M_{n, 1}(\mathscr{R})=p M_{n, 1}(\mathscr{R}) .
$$

Since $\mathscr{M}$ is closed in the weak operator topology, the reverse inclusion also holds:

$$
\mathscr{M}=\overline{\mathscr{M} M_{1, n}(\mathscr{R}) M_{n, 1}(\mathscr{R})} \supseteq \overline{\mathscr{M} M_{1, n}(\mathscr{R})} M_{n, 1}(\mathscr{R})=p M_{n}(\mathscr{R}) M_{n, 1}(\mathscr{R}) .
$$

Hence $\mathscr{M}=p M_{n, 1}(\mathscr{R})=p \mathscr{R}^{n}$.

Finally, suppose that $\mathscr{M}$ is an $\mathscr{R}$-bimodule. We have just proved that $\mathscr{M}=p \mathscr{R}^{n}$ for some projection $p \in M_{n}(\mathscr{R})$, and we must now show that $p \in M_{n}(\mathscr{C})$. Denote by $\mathscr{R}^{(n)}$ the subalgebra of $M_{n}(\mathscr{R})$ consisting of all diagonal matrices with the same element along the diagonal. Since $\mathscr{M}$ is a left $\mathscr{R}$-module, we have $\mathscr{R}^{(n)} \mathscr{M} \subseteq \mathscr{M}$ or (equivalently) $(1-p) \mathscr{R}^{(n)} p \mathscr{R}^{n}=0$. This implies that $(1-p) \mathscr{R}^{(n)} p=0$; hence, $p$ commutes with the von Neumann algebra $\mathscr{R}^{(n)}$. It is well known (and easy to verify) that the commutant of $\mathscr{R}^{(n)}$ is $M_{n}\left(\mathscr{R}^{\prime}\right)$ (where $\mathscr{R}^{\prime}$ is the commutant of $\left.\mathscr{R}\right)$; hence, $p \in M_{n}(\mathscr{R}) \cap M_{n}\left(\mathscr{R}^{\prime}\right)=$ $M_{n}\left(\mathscr{R} \cap \mathscr{R}^{\prime}\right)=M_{n}(\mathscr{C})$.

Proof of Theorem 2.1. We may identify the $C^{*}$-algebra $\mathscr{A}$ with its image under the universal representation on some Hilbert space $\mathscr{H}$. It is well known, then, 
that each bounded linear functional on $\mathscr{A}$ can be uniquely extended to a weakoperator continuous linear functional on the weak-operator closure $\overline{\mathscr{A}}$ of $\mathscr{A}$ and, consequently, that $\overline{\overline{\mathscr{K}}}=\overline{\mathscr{K}} \cap \mathscr{A}$ for each convex subset $\mathscr{K}$ of $\mathscr{A}$ (where one bar denotes the closure in the weak operator topology and two bars denote the norm closure; see [4, p. 713]). It is clear that (if $n$ is finite) these properties hold also for $\mathscr{A}^{n}$ (in place of $\mathscr{A}$ ); in particular, for each $\vec{a} \in \mathscr{A}^{n}$ we have $\overline{\overline{\mathscr{E}} \vec{a}}=\mathscr{A}^{n} \cap \overline{\mathscr{E}} \vec{a}$. Let $\vec{b} \in \mathscr{A}^{n}$ be such that $\vec{b} \notin \overline{\overline{\mathscr{E}} \vec{a}}$. Then $\vec{b} \notin \overline{\mathscr{E} \vec{a}}$. Since $\overline{\mathscr{E} \vec{a}}$ is an $\mathscr{A}$-bimodule in $\mathscr{A}^{n}$, by Lemma 2.2 there exists a projection $p \in M_{n}(\mathscr{C})$ (where $\mathscr{C}$ is the centre of $\overline{\mathscr{A}}$ ) such that $\overline{\mathscr{E}} \vec{a}=p \overline{\mathscr{A}^{n}}$. With $q=1-p$, we now have $q \vec{a}=0$ and $q \vec{b} \neq 0$ (since $\vec{b} \notin \overline{\mathscr{E}} \vec{a}$ ). Thus, if $c_{j} \quad(j=1, \ldots, n)$ are the entries of a suitable row of $q$, then

$$
\sum_{j=1}^{n} c_{j} a_{j}=0, \quad \sum_{j=1}^{n} c_{j} b_{j} \neq 0,
$$

and $c_{j} \in \mathscr{C}$ for each $j$. Now choose any irreducible representation $\pi$ of $\overline{\mathscr{A}}$ such that $\pi\left(\sum_{j=1}^{n} c_{j} b_{j}\right) \neq 0$ (this is possible by elementary $C^{*}$-theory [10, p. 147]). Since the centre of each irreducible algebra consists only of scalar multiples of the identity we have $\pi\left(c_{j}\right)=\lambda_{j} 1$, where $\lambda_{j} \in \mathbb{C}$. Hence relations (2) imply

$$
\sum_{j=1}^{n} \lambda_{j} \pi\left(a_{j}\right)=0 \text { and } \sum_{j=1}^{n} \lambda_{j} \pi\left(b_{j}\right) \neq 0
$$

or

$$
\sum_{j=1}^{n} \lambda_{j} a_{j} \in \operatorname{ker} \pi \cap \mathscr{A} \quad \text { and } \quad \sum_{j=1}^{n} \lambda_{j} b_{j} \notin \operatorname{ker} \pi \cap \mathscr{A} .
$$

Thus, with $\vec{\lambda}=\left(\lambda_{1}, \ldots, \lambda_{n}\right)$, we have $\vec{\lambda} \cdot \vec{b} \notin[\vec{\lambda} \cdot \vec{a}]$. This proves the nontrivial part of the statement in the theorem concerning $C^{*}$-algebras.

The statement concerning von Neumann algebras follows immediately from Lemma 2.2: if $\vec{b} \notin \overrightarrow{\mathscr{C}} \vec{a}$, then there exists a projection $q \in M_{n}(\mathscr{C})$ such that $q \vec{a}=0$ and $q \vec{b} \neq 0$; hence, for some row $\vec{\lambda}$ of $q$ we have $\vec{\lambda} \cdot \vec{a}=0$ and $\vec{\lambda} \cdot \vec{b} \neq 0$.

The following result is an obvious consequence of Theorem 2.1.

Corollary 2.3. Let $\mathscr{A}$ be a $C^{*}$-algebra and $\mathscr{B}(\mathscr{A})$ the algebra of all bounded linear operators on $\mathscr{A}$. Then the closure in the strong operator topology (= pointnorm topology) of the algebra $\mathscr{E}$ of all elementary operators on $\mathscr{A}$ (as a subset of $\mathscr{B}(\mathscr{A})$ ) consists precisely of operators $\varphi \in \mathscr{B}(\mathscr{A})$ that satisfy $\varphi(\mathscr{K}) \subseteq \mathscr{K}$ for each closed ideal $\mathscr{K}$ in $\mathscr{A}$.

If $\mathscr{A}$ is a von Neumann algebra, then $\mathscr{E}$ is a dense subset of the space of all bounded module endomorphisms of $\mathscr{A}$ over the centre $\mathscr{C}$ equipped with the point-weak operator topology. (Here the point-weak operator topology on $\mathscr{B}(\mathscr{A})$ is defined by the family of seminorms $\psi \rightarrow|\langle\psi(a) \xi, \eta\rangle|$, where $\xi$ and $\eta$ are arbitrary vectors from the Hilbert space on which $\mathscr{A}$ is acting.)

An operator $\varphi \in \mathscr{B}(\mathscr{A})$ is called a local elementary operator if for each $x \in \mathscr{A}$ there exists an elementary operator $E$ (depending on $x$ ) such that 
$\varphi x=E x$. Larson and Sourour observed in [5] that for each infinite-dimensional Banach space $\mathscr{Z}$ there exist on $\mathscr{B}(\mathscr{X})$ nonelementary local elementary operators. Corollary 2.3 implies that on $C^{*}$-algebras local elementary operators can be strongly approximated by elementary operators.

To study the range inclusion problem for elementary operators, we shall need a sharper form of Proposition 1.1 for factors, but to prove it, we need a lemma.

Lemma 2.4. Let $\mathscr{A}$ be a unital prime $C^{*}$-algebra (or, more generally, a unital complex ultraprime Banach algebra in the sense [9]), $\mathscr{E}$ the algebra of all elementary operators on $\mathscr{A}, \mathscr{S}$ a finite-dimensional subspace of $\mathscr{A}$, and $b \in \mathscr{A} \backslash \mathscr{S}$. Then there exists $E \in \mathscr{E}$ such that $E \mathscr{S}=0$ and $E b \neq 0$.

Proof. The proof is by an induction on the dimension $n$ of $\mathscr{S}$. First assume that $n=1$, and choose a nonzero element $a \in \mathscr{S}$. If for each $E \in \mathscr{E}$ the condition $E a=0$ implies $E b=0$, then the correspondence $E a \rightarrow E b$ is a well-defined $\mathscr{A}$-bimodule homomorphism from the ideal $\mathscr{E} a$ to $\mathscr{A}$, which maps $a$ to $b$. By [7, Proposition 2.5] (or, if $\mathscr{A}$ is a general ultraprime Banach algebra, by [9, Theorem 4.1]) each such homomorphism is necessarily a multiplication by certain $\gamma \in \mathbb{C}$. Hence we have $b=\gamma a$; but this is in contradiction with $b \notin \mathscr{S}$. Hence there exists an $E \in \mathscr{E}$ such that $E a=0$ and $E b \neq 0$.

Now let $n$ be any positive integer, assume inductively that the lemma holds for all subspaces of dimension at most $n$, and let $\mathscr{S}$ be an arbitrary $(n+1)$ dimensional subspace of $\mathscr{A}$. Choose a basis $\left\{a_{1}, \ldots, a_{n}, a\right\}$ for $\mathscr{S}$ and denote by $\mathscr{T}$ the span of $\left\{a_{1}, \ldots, a_{n}\right\}$. By the inductive hypothesis there exists $F \in \mathscr{E}$ such that $F \mathscr{T}=0$ and $F b \neq 0$. If $F b \notin \mathbb{C} F a$, then (by the already proved case $n=1$ ) there exists $G \in \mathscr{E}$ such that $G F a=0$ and $G F b \neq 0$; hence, $E \stackrel{\text { def }}{=} G F$ satisfies $E \mathscr{S}=0$ and $E b \neq 0$. Thus we may assume that $F b=\alpha F a$ for some $\alpha \in \mathbb{C}$. Put $c=b-\alpha a$ and note that $F c=0$. Then $c \notin \mathscr{T}$ (since $b \notin \mathscr{S}$ ). Hence by the inductive hypothesis there exists $H \in \mathscr{E}$ such that $H \mathscr{T}=0$ and $H c \neq 0$. Since $\mathscr{A}$ is a prime algebra and $F a \neq 0, H c \neq 0$, there exists $d \in \mathscr{A}$ such that $F(a) d H(c) \neq 0$. Finally, let $E \in \mathscr{E}$ be defined by

$$
E x=-F(x) d H(a)+F(a) d H(x) \quad(x \in \mathscr{A}) .
$$

Then $E a=0, E \mathscr{T}=0$ (since $F \mathscr{T}=0$ and $H \mathscr{T}=0$ ), and

$$
E b=E(c+\alpha a)=E c=-F(c) d H(a)+F(a) d H(c)=F(a) d H(c) \neq 0 .
$$

In the proof of our last two results we shall also use the following observation: if $f_{k} \quad(k=1,2, \ldots)$ is an increasing sequence of projections in a factor $\mathscr{A}$ and $e \in \mathscr{A}$ is a projection such that $f_{k} \prec e$ for each $k$, then $f \preceq e$, where $f=\bigvee_{k=1}^{\infty} f_{k}$. If all projections $f_{k}$ are finite, this follows from [12, Lemma $2.2, \mathrm{p} .310]$. If some $f_{k}$ is infinite, then the observation follows from the fact that any two infinite cyclic projections in a factor are equivalent [4, p. 414], by noting that the cardinal number of cyclic summands in $f$ is less than or equal to the cardinal number of cyclic summands in $e$ (since $f=f_{1}+\sum_{k=1}^{\infty}\left(f_{k+1}-f_{k}\right)$ and $\left.f_{k+1}-f_{k} \prec e, f_{1} \prec e\right)$.

Proposition 2.5. Let $\mathscr{A}$ be a von Neumann factor, $\mathscr{K}$ a closed ideal in $\mathscr{A}$, and $a_{1}, \ldots, a_{n}$ elements of $\mathscr{A}$ linearly independent modulo $\mathscr{K}$. Then there exist ideals $\mathscr{I}_{j}(j=1, \ldots, n)$ in $\mathscr{A}$ such that $\mathscr{I}_{j} \supset \mathscr{K}$ for each $j$ (where the 
symbol $\supset$ is used in the strict sense; that is, $\left.\mathscr{I}_{j} \neq \mathscr{K}\right)$ and

$$
\mathscr{E} \vec{a} \supseteq \bigoplus_{j=1}^{n} \mathscr{I}_{j},
$$

where $\vec{a}=\left(a_{1}, \ldots, a_{n}\right)$.

Proof. The proof is again by an induction on $n$. In the case $n=1$ it suffices to prove that the ideal $\left\langle a_{1}\right\rangle$ generated by $a_{1}$ contains $\mathscr{K}$. Since $a_{1} \notin \mathscr{K}$, we see (using the polar decomposition of $a_{1}$ and the spectral theorem) that the spectral projection $e$ of $\left|a_{1}\right|$ corresponding to a certain positive interval satisfies $e \notin \mathscr{K}$ and $e \in\left\langle a_{1}\right\rangle$. Hence it suffices to prove that $\mathscr{K} \subseteq\langle e\rangle$. For this, it suffices to show that $b \in\langle e\rangle$ for each positive $b$ in $\mathscr{K}$. For each $k=1,2, \ldots$, let $f_{k}$ be the spectral projection of $b$ corresponding to the interval $[1 / k,\|b\|]$. Then $f \stackrel{\text { def }}{=} \bigvee_{k=1}^{\infty} f_{k}$ is the range projection of $b$; hence, $b=f b$. From $f_{k} \in \mathscr{K}, e \notin \mathscr{K}$, and the fact that any two projections in a factor are comparable [4, p. 408], it follows that $f_{k} \prec e$ for each $k$; hence, $f \preceq e$. This implies that $f \in\langle e\rangle$; therefore, we have $b=f b \in\langle e\rangle$.

Now let $n>1$. If we can prove that there exists an $E \in \mathscr{E}$ such that

$$
E a_{j}=0 \text { for } j=1, \ldots, n-1 \text { and } E a_{n} \notin \mathscr{K},
$$

then by a previous paragraph (applied to $E a_{n}$ ) we have

$$
\mathscr{E} \vec{a} \supseteq \mathscr{E}\left(0, \ldots, 0, E a_{n}\right)=0 \oplus \cdots \oplus 0 \oplus\left\langle E a_{n}\right\rangle \supset 0 \oplus \cdots \oplus 0 \oplus \mathscr{K},
$$

and by applying the same arguments also to other components, the proposition follows. To prove (4), assume, to the contrary, that the condition $E a_{j}=0$ for $j=1, \ldots, n-1$ implies that $E a_{n} \in \mathscr{K}$. Then the map

$$
\begin{gathered}
\varphi: \mathscr{E}\left(a_{1}, \ldots, a_{n-1}\right) \rightarrow \mathscr{A} / \mathscr{K}, \\
\varphi\left(E a_{1}, \ldots, E a_{n-1}\right) \stackrel{\text { def }}{=} E a_{n}+\mathscr{K} \quad(E \in \mathscr{E})
\end{gathered}
$$

is a well-defined homomorphism of $\mathscr{E}$-modules. By the inductive hypothesis we have $\mathscr{E}\left(a_{1}, \ldots, a_{n-1}\right) \supseteq \mathscr{K}^{n-1}$. The components $\varphi_{j}$ of the restriction of $\varphi$ to $\mathscr{K}^{n-1}$ are $\mathscr{E}$-module homomorphisms from $\mathscr{K}$ to $\mathscr{A} / \mathscr{K}$; hence, $\varphi_{j}=0$ for each $j=1, \ldots, n-1$ (since $\varphi_{j}(\mathscr{K})=\varphi_{j}(\mathscr{K} \mathscr{K})=\mathscr{K} \varphi_{j}(\mathscr{K})=0$ in $\mathscr{A} / \mathscr{K})$. It follows that $\varphi\left(\mathscr{K}^{n-1}\right)=0$; hence, $\varphi$ induces an $\mathscr{E}$-module homomorphism $\tilde{\varphi}: \mathscr{E}\left(a_{1}, \ldots, a_{n-1}\right) / \mathscr{K}^{n-1} \rightarrow \mathscr{A} / \mathscr{K}$. Identifying in the obvious way $\mathscr{E}\left(a_{1}, \ldots, a_{n-1}\right) / \mathscr{K}^{n-1}$ with $\tilde{\mathscr{E}}\left(a_{1}+\mathscr{K}, \ldots, a_{n-1}+\mathscr{K}\right)$, where $\tilde{\mathscr{E}}$ denotes the algebra of all elementary operators on $\mathscr{A} / \mathscr{K}$, we can regard $\tilde{\varphi}$ as an $\widetilde{\mathscr{E}}$-module homomorphism from $\tilde{\mathscr{E}}\left(a_{1}+\mathscr{K}, \ldots, a_{n-1}+\mathscr{K}\right)$ to $\mathscr{A} / \mathscr{K}$ such that $\tilde{\varphi}\left(\widetilde{E}\left(a_{1}+\mathscr{K}\right), \ldots, \widetilde{E}\left(a_{n-1}+\mathscr{K}\right)\right)=\widetilde{E}\left(a_{n}+\mathscr{K}\right)(\widetilde{E} \in \widetilde{\mathscr{E}})$. In particular, for any fixed $\widetilde{E} \in \tilde{\mathscr{E}}$ we have $\widetilde{E}\left(a_{n}+\mathscr{K}\right)=0$ if $\widetilde{E}\left(a_{j}+\mathscr{K}\right)=0$ for all $j=1, \ldots, n-1$. Hence by Lemma $2.4 a_{n}+\mathscr{K}$ must be contained in the subspace of $\mathscr{A} / \mathscr{K}$ spanned by $\left\{a_{j}+\mathscr{K}: j=1, \ldots, n-1\right\}$, but this is in contradiction with the assumed linear independence modulo $\mathscr{K}$ of elements $a_{j} \quad(j=1, \ldots, n)$. (Here we have used the fact that $\mathscr{A} / \mathscr{K}$ is a prime $C^{*}$ algebra, since the closed ideals in a factor are linearly ordered by inclusion [4, p. 451].) 
Proposition 2.5 can be useful in studying the question of when the range of a fixed elementary operator is contained in a given (not necessarily closed) ideal $\mathscr{I}$ of $\mathscr{A}$. In the special case $\mathscr{I}=0$, Mathieu [7, 9] solved the problem for general prime $C^{*}$-algebras and ultraprime Banach algebras. In the case $\mathscr{A}=\mathscr{B}(\mathscr{H})$ the question was studied in $[3,1,2,6]$; in particular, the case $\mathscr{A}=\mathscr{B}(\mathscr{H})$ of the following corollary was proved by Apostol and Fialkow [1, Theorem 3.1].

Corollary 2.6. Let $\mathscr{I}$ be a (not necessarily closed) ideal in a factor $\mathscr{A}$, and let $E$ be an elementary operator on $\mathscr{A}$ defined by

$$
E x=\sum_{j=1}^{n} a_{j} x b_{j} \quad(x \in \mathscr{A}),
$$

where $\vec{a}=\left(a_{1}, \ldots, a_{n}\right)$ and $\vec{b}=\left(b_{1}, \ldots, b_{n}\right)$ are fixed elements in $\mathscr{A}^{n}$. If $E(\mathscr{A}) \subseteq \mathscr{I}$ and $a_{1}, \ldots, a_{n}$ are linearly independent modulo the norm closure $\overline{\bar{I}}$ of $\mathscr{I}$ then each $b_{j}(j=1, \ldots, n)$ must be in $\mathscr{I}$.

Proof. We shall prove that $b_{1} \in \mathscr{I}$; the proof that $b_{j} \in \mathscr{I}$ for $j>1$ is the same. By Proposition 2.5 there exists an elementary operator $F$, say

$$
F x=\sum_{i=1}^{m} u_{i} x v_{i} \quad(x \in \mathscr{A}),
$$

where $u_{j}, v_{j} \in \mathscr{A}$ are fixed, such that

$$
F a_{j}=0 \text { for } j=2, \ldots, n \text { and } F a_{1} \notin \overline{\overline{\mathscr{I}}} \text {. }
$$

Since by hypothesis $E(\mathscr{A}) \subseteq \mathscr{I}$ and $\mathscr{I}$ is an ideal, we have $\sum_{i=1}^{m} u_{i} E\left(v_{i} x\right) \in$ $\mathscr{I}$ for all $x \in \mathscr{A}$, which can be written as

$$
\sum_{i=1}^{m} u_{i} \sum_{j=1}^{n} a_{j} v_{i} x b_{j} \in \mathscr{I} \quad(x \in \mathscr{A}) .
$$

Reversing the order of summation and using (5) we obtain that $\left(F a_{1}\right) x b_{1} \in \mathscr{J}$ for all $x \in \mathscr{A}$. Denoting $F a_{1}$ by $a$ and $b_{1}$ by $b$, we now have

$$
a \mathscr{A} b \subseteq \mathscr{I} \text { and } a \notin \overline{\overline{\mathscr{I}}} \text {, }
$$

and we must prove that this implies that $b \in \mathscr{I}$.

Using the polar decomposition of $a$ and $b^{*}$ and the spectral theorem for $|a|=\sqrt{a^{*} a}$ it follows from (6) by classical arguments (see $[4, \S 6.8]$ ) that for some spectral projection $e$ of $|a|$ we have

$$
e \mathscr{A}\left|b^{*}\right| \subseteq \mathscr{I} \text { and } e \notin \overline{\bar{I}} \text {. }
$$

For each $k=1,2, \ldots$ let $f_{k}$ be the spectral projection of $\left|b^{*}\right|$ corresponding to $(1 / k, \infty)$. Since any two projections in a factor are comparable [4, p. 408], there are now two cases: (i) $e \preceq f_{k}$ for some positive integer $k$; (ii) $f_{k} \prec e$ for each $k$; but, from $e \mathscr{A}\left|b^{*}\right| \subseteq \mathscr{I}$ we have $e \mathscr{A} f_{k} \subseteq \mathscr{I}$ for each $k$. Hence in the first case it follows that $e \in \mathscr{I}$ (since $e=e u f_{k} u^{*} \in \mathscr{I} u^{*} \subseteq \mathscr{I}$, where $u \in \mathscr{A}$ is a partial isometry with initial projection contained in $f_{k}$ and final projection $e$ ), which is in contradiction with $e \notin \overline{\bar{I}}$. Thus, only case (ii) occurs. Since 
$f_{k} \prec e$ for all $k$, the range projection $f=\bigvee_{k=1}^{\infty} f_{k}$ of $\left|b^{*}\right|$ satisfies $f \preceq e$. Denoting by $v$ a partial isometry with initial projection $f$ and final projection contained in $e$, we have $\left|b^{*}\right|=f\left|b^{*}\right|=v^{*} e v\left|b^{*}\right| \in \mathscr{I}\left(\right.$ since $\left.e \mathscr{A}\left|b^{*}\right| \subseteq \mathscr{I}\right)$. Hence $b \in \mathscr{I}$ by polar decomposition.

\section{REFERENCES}

1. C. Apostol and L. Fialkow, Structural properties of elementary operators, Canad. J. Math. 38 (1986), 1485-1524.

2. L. Fialkow, The range inclusion problem for elementary operators, Michigan Math. J. 34 (1987), 451-459.

3. C. K. Fong and A. R. Sourour, On the operator identity $\sum A_{k} X B_{k}=0$, Canad. J. Math. 31 (1979), 845-857.

4. R. V. Kadison and J. R. Ringrose, Fundamentals of the theory of operator algebras, vols. I and II, Academic Press, London, 1983, 1986.

5. D. R. Larson and A. R. Sourour, Local derivations and local automorphisms of $\mathscr{B}(\mathscr{X})$, Proc. Sympos. Pure Math., vol. 51, part 2, Amer. Math. Soc., Providence, RI, 1990, pp. 187-194.

6. B. Magajna, A system of operator equations, Canad. Math. Bull. 'j0 (1987), 200-209.

7. M. Mathieu, Elementary operators on prime $C^{*}$-algebras. I, Math. Ann. 284 (1989), 223244.

8. _ Applications of ultraprime Banach algebras in the theory of elementary operators, Thesis, Tübingen, 1986.

9. __ Rings of quotients of ultraprime Banach algebras, with applications to elementary operators, Proc. Centre Math. Anal. Austral. Nat. Univ. 21 (1989), 297-317.

10. G. J. Murphy, $C^{*}$-algebras and operator theory, Academic Press, London, 1990.

11. R. S. Pierce, Associative algebras, Graduate Texts in Math., vol. 88, Springer-Verlag, New York, 1982.

12. M. Takesaki, Theory of operator algebras. I, Springer-Verlag, New York, 1979.

Department of Mathematics, University of Ljubljana, JadRanska 19, Ljubljana 61000 , SLOVENIJA

E-mail address: Bojan.Magajna@uni-lj.si 